\title{
The Influence of Residual Vertical Dispersion on LEP Performance
}

\author{
P.Collier \& H. Schmickler, CERN, 1211-Geneva, Switzerland
}

\begin{abstract}
During LEP Operation for Luminosity production, the closed orbit is systematically corrected towards a reference orbit which has been empirically found to produce high luminosities. Machine studies have been undertaken to try and understand the mechanism by which the vertical closed orbit affects the luminosity. The dominantparameter has been found to be the residual vertical dispersion, in particular the residual vertical dispersion at the interaction points. This paper reports on studies to investigate how the quality of the closed orbit affects the residual vertical dispersion and especially the residual vertical dispersion at the interaction points.
\end{abstract}

\section{INTRODUCTION}

During the LEP operation period in 1994, the operations group found that the luminosity performance of LEP depended critically on the vertical closed orbit [1]. By reloading orbit correctors from a previous 'good' physics fill and correcting back to the orbit measured during this fill, reproducibly good performance for the machine was attained.

Work was started to try and understand the link between orbit and luminosity performance. The vertical orbit in LEP is routinely corrected to a sigma of about $0.6 \mathrm{~mm}$. It was quickly found that 'good' and 'bad' orbits for luminosity were qualitatively the same, in terms of sigma's. The difference between the two being more related to the history of orbit corrections used to achieve the desired result, than the global features of the orbit itself.

The studies made in 1994 concentrated on three main beam parameters which could be affected by the correction of the vertical closed orbit; average residual vertical dispersion, coupling and the localresidual dispersion at the interaction points.

\section{Average Residual Vertical Dispersion}

The average residual vertical dispersion is an important parameter which can lead to significant increases in beam size and hence, lower luminosity. It is also known to be driven by the specific details of how the orbit is corrected. For example, the correction of the effect of magnet misalignments with nonlocal orbit correctors and asymmetric bumps across the interaction regions are all known to drive the vertical dispersion. A systematic series of measurements of the dispersion were taken during physics fills. The measured sigma of the residual dispersions were all in the range of 5 to $15 \mathrm{~cm}$. No correlation with the luminosity could be found [2]. A dedicated study of the variation of vertical beam size with dispersion was undertaken. The results are shown in figure 1. For values below $15 \mathrm{~cm}$, the residual vertical dispersion no longer dominates as the source of increased beam size. Thus, the luminosity performance of LEP in physics cannot be explained by vertical dispersion alone.

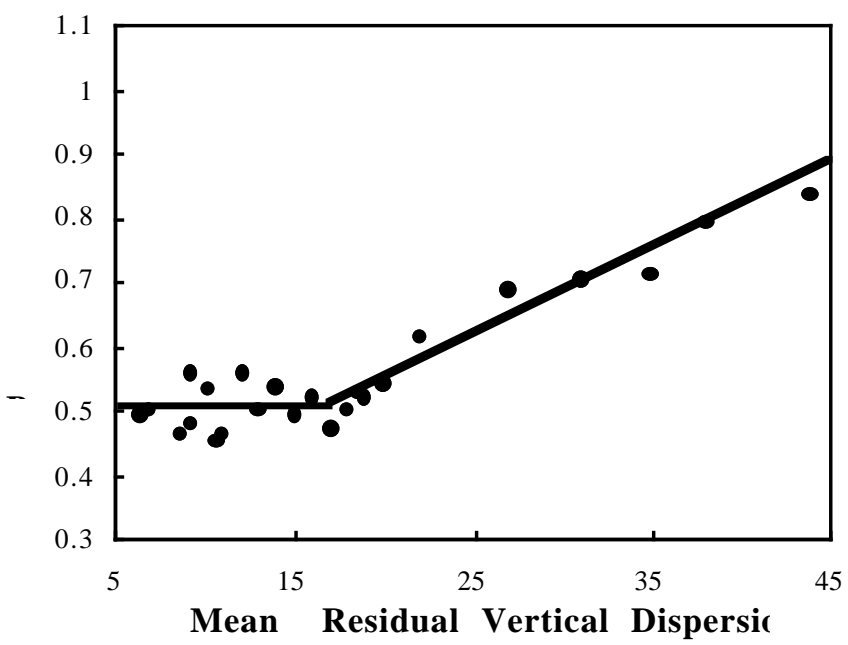

Figure 1: Vertical Beam Size, Measured using a UV monitor in LEP as a Function of rms. Vertical Dispersion

\section{Coupling}

Coupling can be driven by discrete sources, such as the experimental solenoids (known as solenoid coupling) and by extended sources, such as the closed orbit in the sextupoles (machine coupling). The latter case could provide the mechanism whereby the verticalclosed orbit affects the luminosity performance of LEP. In both cases, the correction of coupling in LEP is made with discrete sqew quadrupoles installed in the straight sections around each LEP experiment.

Studies on the effect of coupling on the luminosity performance have been published [2]. It was found that the machine coupling, characterized by the closest tune approach method, could be changed significantly by the strategy used to correct the vertical closed orbit. The emittance ratio, $\kappa$, generated by a given value of coupling is related to the closest tune approach by the equation [3]:

where

$$
\kappa=1-\frac{\left(2 r^{2}+1\right)}{\left(4 r^{2}+1\right)}
$$

$$
r=\frac{\delta q}{2\left|q_{h}-q_{v}\right|}
$$

Where, $\left|q_{h}-q_{v}\right|$ is the distance of the working point to the main coupling resonance and $\delta q$ is the measured closest tune approach. In LEP $\left|\mathrm{fq}_{\mathrm{v}}\right|=0.14$. For high luminosity performance the emittance ratio must be kept below the ratio of the betatron functions at the interaction point. In LEP the betatron function ratio is $2 \% \quad\left(\beta_{h}^{*}=2 \mathrm{~m}, \beta^{*}{ }_{v}=5 \mathrm{~cm}\right)$. For 
this, a closest tune approach of less than 0.028 is acceptable. It was found that, for all correction strategies tested, the measured closest tune approach value was less than this limit.

It was also speculated that the first synchrotron sideband of the main coupling resonance would have a similar effect to the main coupling resonance. Theoretical studies made since[4], have shown that the synchrotron sideband of the main coupling resonance is not strong enough to significantly affect the emittance ratio, and hence the luminosity performance. However, the coupling remains a parameter which is routinely adjusted by the operators, in order to achieve the highest performance. If the machine is tuned to the highest performance, especially at the end of a physics coast, the emittance ratio is well below the $2 \%$ limit mentioned above. For these conditions the tuning constraints for coupling become more strict and a closest tune approach of the order of 0.01 has to be achieved.

\section{OFFSETS FROM K-MODULATION}

The vertical closed orbit is of critical importance for generating polarized beams in LEP. Polarization is used routinely to measure the LEP beam energy with high precision [5]. In order to improve the quality of the orbit correction and hence polarization levels a system was installed to allow a measurement of the offset of the axis of the orbit pickups to the center of the quadrupole next to it. The system makes use of the technique called k-modulation. So far it has been installed on some of the insertion quadrupoles near to each of the interaction points.

The technique makes use of a low-frequency generator to modulate the strength of a selected quadrupole [6,7]. A sensitive pickup is used to detect the amplitude of the resulting oscillations of the beam at the excitation frequency. The amplitude of the oscillations at the pickup is related to the beam position in the quadrupole which is being modulated and is a minimum when the beam passes through the magnetic center of the quadrupole. Figure 2 shows the amplitude of the beam motion at the k-modulation frequency for various positions of the beam in the modulated quadrupole. The curve has its minimum at $0.13 \mathrm{~mm}$, which is interpreted as an offset between the pickup 'zero' reading and the magnetic center of the quadrupole. All offsets determined this way (the values attain numbers as large as $2 \mathrm{~mm}$ ) are introduced as additional calibration constants into the orbit measurement system.

Offsets have been measured for several pickups around one of the LEP interaction points and compared with pickup readings taken from a 'golden' orbit, producing high luminosity performance. The data is shown in figure 3 . Here the pickup reading for the 'golden' orbit are shown (hatched) together with the same readings after correction for the offsets measured using the k-modulation technique (solid). From the data of figure 3, it seems likely that the 'golden' orbit is just an orbit where the beams pass close to the center of the insertion quadrupoles. This was tested by introducing the offset data, found by k-modulation, into the orbit measurement package. Various techniques were then use to correct the orbit.
Without the offsets included, the results, in terms of the performance of the machine, tended to be highly variable. With the offsets in place good luminosity conditions were more easily achieved.

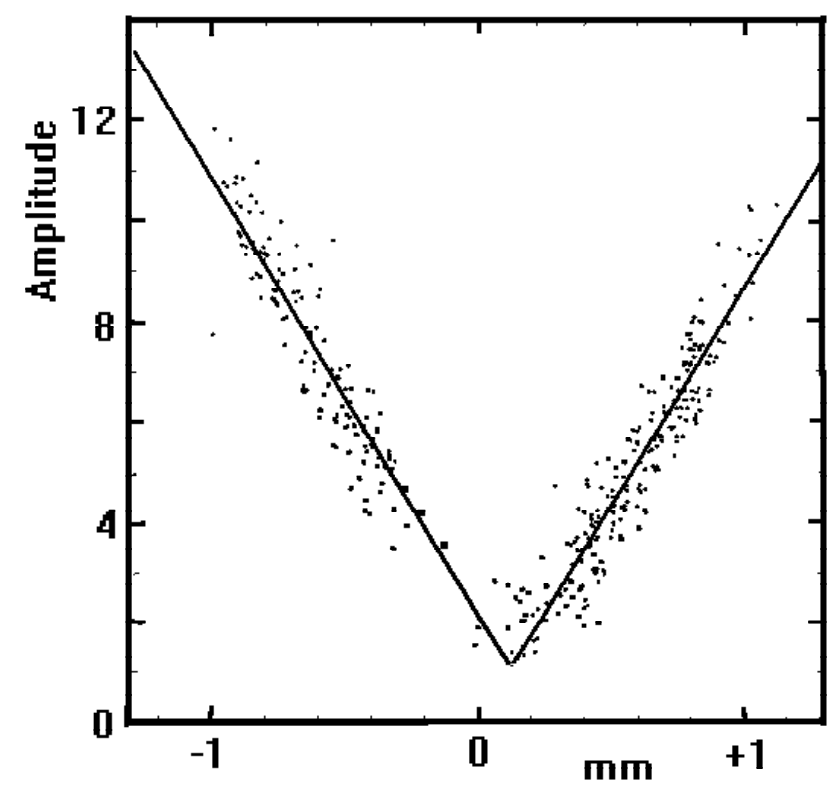

Figure 2: Beam Oscillation Amplitude at a Sensitive Coupler From Modulation of the Strength of a Quadrupole vs. Pickup Reading from the Pickup Next to the Quadrupole.

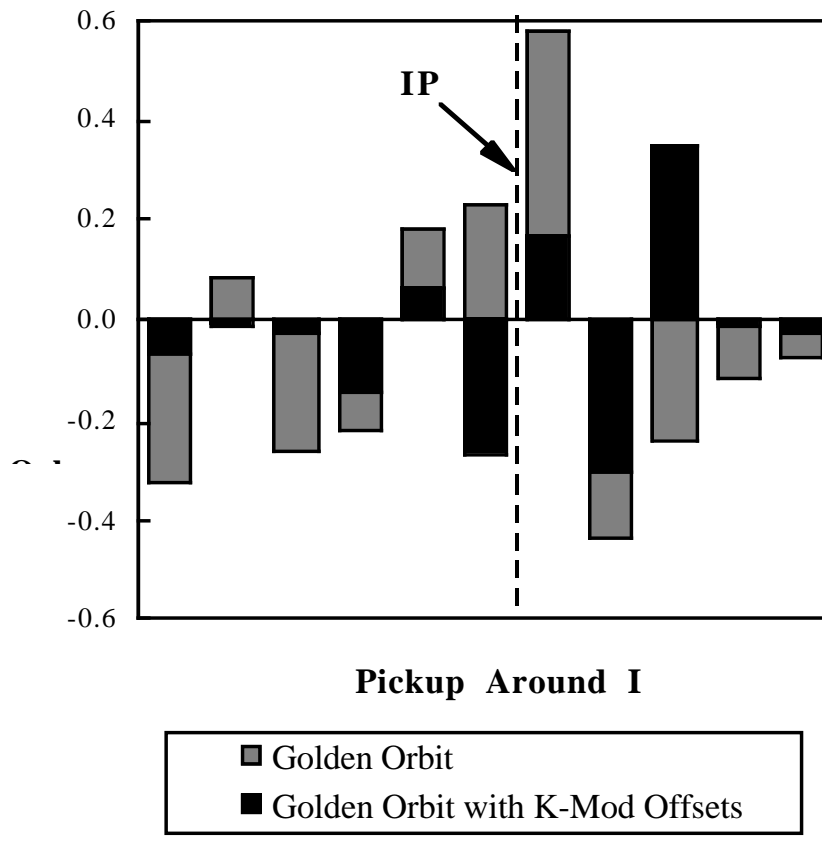

Figure 3: Orbit Readings for a 'Golden Orbit' and the same readings after correction for the offsets measured using kmodulation. 


\section{THE EFFECT OF BEAM OFFSETS IN THE INSERTION QUADRUPOLES ON LUMINOSITY}

From the above chapter it becomes clear that high luminosity performance is achieved by steering the beam through the center of the insertion quadrupoles.

If the beam does not pass through the center of these quadrupoles, the beam experiences a dipole kick. The effect can be corrected with an orbit corrector dipole close by. However, a local dispersion bump in the interaction point can also be produced. This affect has been simulated using the MAD package[8]. Here a $1 \mathrm{~mm}$ offset was introduced into two symmetric quadrupoles next to an interaction point. The resulting simulated orbit was corrected and the dispersion computed. The results gave a dispersion of around $1 \mathrm{~cm}$ at the interaction point.

Any dispersion will contribute to the beam size by quadratic addition with the normal beam size from the emittance, using the relation:

$$
\left(\sigma_{y}^{*}\right)^{2}=\varepsilon_{y} \beta_{y}^{*}+\left(\sigma_{e} D_{y}^{*}\right)^{2}
$$

ie.

$$
\left(\sigma_{y}^{*}\right)^{2}=\left(\sigma_{o}^{*}\right)^{2}+\left(\sigma_{D}^{*}\right)^{2}
$$

Where $\sigma_{\mathrm{y}}{ }^{*}, \mathrm{D}^{\mathrm{y}^{*}}$ and $\beta_{\mathrm{y}}{ }^{*}$ are the beam size, the vertical dispersion and the beta function value at the interaction point, and $\sigma_{\mathrm{e}}$ is the energy spread of the beam. Table 1 shows the effect of a $1 \mathrm{~mm}$ local dispersion bump at one of the LEP interaction points with data presented for typical conditions at the beginning and the end of a LEP physics coast.

Table 1: Typical Performance Reductions Expected from the Introduction of $1 \mathrm{~cm}$ of Vertical Dispersion at one Interaction Point.

\begin{tabular}{|l|c|c|c|}
\hline Conditions & $\sigma_{\mathrm{o}} / \mu \mathrm{m}$ & $\sigma_{\mathrm{D}} / \mu \mathrm{m}$ & $\begin{array}{c}\text { Luminosity } \\
\text { Change } / \%\end{array}$ \\
\hline $\begin{array}{l}\text { Start of Coast : } \\
\varepsilon_{\mathrm{v}} \sim 0.6 \mathrm{~nm} \\
\text { (Full Wigglers) }\end{array}$ & $\mathbf{4 . 0}$ & $\mathbf{1 . 6}$ & $\mathbf{- 8}$ \\
\hline $\begin{array}{l}\text { End of Coast : } \\
\varepsilon_{\mathrm{v}} \sim 0.3 \mathrm{~nm} \\
\text { (Wigglers Off) }\end{array}$ & $\mathbf{2 . 0}$ & $\mathbf{0 . 9}$ & $-\mathbf{1 0}$ \\
\hline
\end{tabular}

With the symmetry of the LEP machine, the largest effect on vertical dispersion is not in the interaction point where the beam is mis-centered, but in the two adjacent interaction points. Hence all possible offsets in all four interaction points interfere and can increase the above effect.

\section{CONCLUSIONS}

Several parameters are affected by the vertical closed orbit correction strategy used during physics coasts. A good control of the coupling compensation and mean residual vertical dispersion are the necessary requisites to obtain high beam- beam tune shifts, above $\xi_{\mathrm{y}}=0.03$. At this level of tuning, further improvements also need the control of the local dispersion in the interaction points. This dispersion is generated by the beam not passing through the magnetic center of the insertion quadrupoles, due to misalignments of the orbit monitors used for beam steering with respect to the magnetic center of the quadrupoles. Simulations of offsets in a single quadrupole have indicated that such offsets reduce the luminosity performance of LEP significantly. The use of a 'golden' orbit by the LEP operations group has empirically solved the problem of these offsets. This type of reference orbit steers the beam close to the center of the insertion quadrupoles. The offsets have been introduced into the closed orbit measurement system and reproducibly good conditions have proved more easy to obtain. During the next LEP running period, more pickup offsets are planned to be measured to further reduce the problem.

\section{REFERENCES}

[1] Bailey, R. et. al., "Operational Procedures to Obtain High Beam-Beam Tune Shifts in LEP Pretzel Operation", This Conference.

[2] Collier, P. \& Schmickler, H., "The Influence of the Vertical Closed Orbit on Luminosity Performance in LEP”, Proceedings, EPAC-94, London, U.K., 1994.

[3] Guignard, G., "Adjustment of Emittance Ratio by Coupling in e-p Storage Rings", Proceedings, XI High Energy Accelerator Conference, CERN, Geneva, CH., 1980.

[4] Alexahin, Y.I., "On the Strength of the Synchrotron Sideband of the Main Coupling resonance in LEP", SLNote 94-103 (AP), CERN, CH., 1994.

[5] Kouchouk, J.P. et al., "Energy Calibration with a Polarized Beam at LEP”, Proceedings, XVI Conference on High Energy Accelerators, Hamburg, Germany, 1992.

[6] Reichel, I., "Beam Position Measurement by Modulation of Quadrupole Strengths", Diploma Thesis, RWTH of Aachen, PITHA 94154, 1995.

[7] Barnett, I. et al., "Dynamic Beam Based Alignment", SL Note 94-84 (BI), CERN, Switzerland, 1994.

[8] Verdier, A., CERN, Switzerland, private communication. 